\title{
Flagellum Degeneration in the Fungus Phytophthora palmivora (formerly Phytophthora parasitica)
}

\author{
By D. E. HEMMES AND H. R. HOHL \\ Cytological Laboratory of the Institute of General Botany, \\ University of Zurich, Zurich, Switzerland
}

(Received 6 April 1972; revised I4 June 1972)

\section{SUMMARY}

Direct sporangial germination in Phytophthora palmivora (formerly Phytophthora parasitica) is accompanied by a regular series of events leading to the complete breakdown of all flagella present in the mature sporangia. Electron microscopy of thin sections shows that the breakdown involves the cleavage of the ensheathing flagellar vacuoles into small vesicles, thus leaving the axonemes unenclosed in the cytoplasm. In a second stage the axonemal microtubules lose their parallel arrangement and gradually disintegrate. The central microtubules break down first and thus appear to be more fragile than the peripheral ones.

\section{INTRODUCTION}

Sporangia of the phycomycete Phytophthora palmivora are able to germinate with zoospores (indirect germination) or with germ tubes (direct germination), yet in both instances flagella are formed first (Hemmes \& Hohl, 1969). The flagella serve as locomotive organs for zoospores during indirect germination, but they have no apparent function during direct germination. In fact, the flagella degenerate before germ-tube formation. The breakdown occurs quite synchronously during a period of about $I h$, starting $I h$ after the sporangia have been submersed into a medium favouring direct germination.

We have studied the ultrastructural aspects of the sequence of flagellar degeneration and describe in this report the cleavage of the flagellar vacuole and the subsequent dissolution of the axoneme.

\section{METHODS}

Phytophthora palmivora Butler (an isolate formerly referred to as Phytophthora parasitica PI13, see Tokunaga \& Bartnicki-Garcia, 1971) was used throughout this study. It represents a single-spore isolate and has the advantage that sporangial formation can be induced by exposing the dark-grown mycelium to light (Aragaki \& Hine, 1963).

The fungus was grown in darkness at $32{ }^{\circ} \mathrm{C}$ on a vegetable-juice agar (10\% V-8 juice (Campbell Soup Corporation, Felegara (Parma), Italy), $0.1 \% \mathrm{CaCO}_{3}, 1.5 \%$ agar) in $90 \times$ I $5 \mathrm{~mm}$ Petri dishes. After 3 to 4 days the cultures were placed in fluorescent light with the temperature of the plates approximately $28{ }^{\circ} \mathrm{C}$.

Petri dishes, heavily covered with sporangia, were flooded with $5 \mathrm{ml}$ of filtered $8 \%$ aqueous solutions of dextrin at $28^{\circ} \mathrm{C}$. Submerged sporangia were then brushed loose from the hyphae with a transferring loop and the resulting suspensions incubated at $28^{\circ} \mathrm{C}$ in $60 \times 15 \mathrm{~mm}$ plastic Petri dishes in which sporangia sank and adhered to the bottom of the dish. Under these conditions the sporangia germinated with germ tubes after a period of $3 \mathrm{~h}$. 

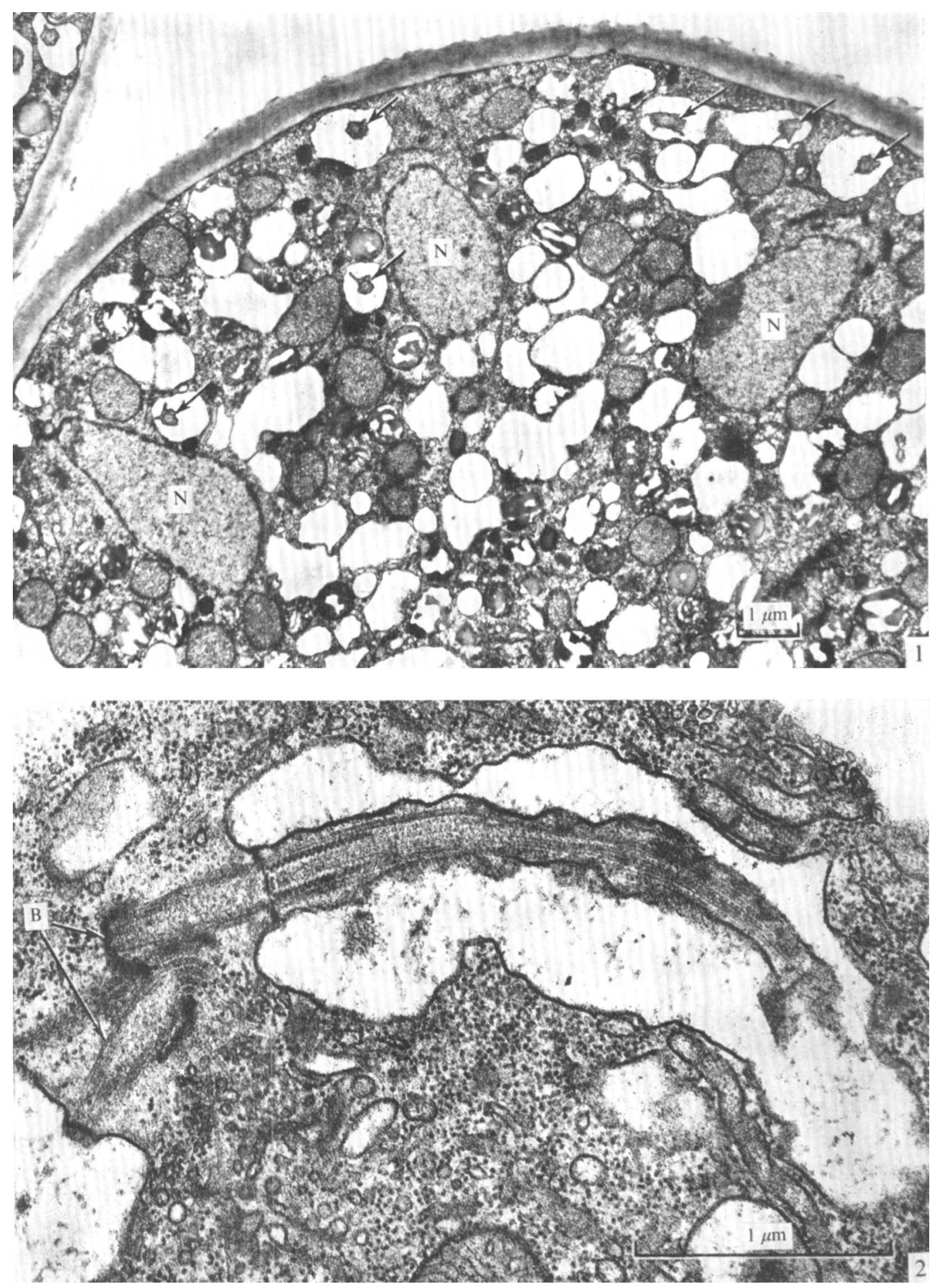

Fig. I. Representative portion of sporangium fixed directly on the hyphae showing the distribution and orientation of nuclei and pattern of flagella within vacuoles at the sporangial periphery. Nuclei $(\mathrm{N})$; flagella (arrows).

Fig. 2. Section through paired basal bodies (B) showing the projection of the flagellar axoneme into the flagellar vacuole. 

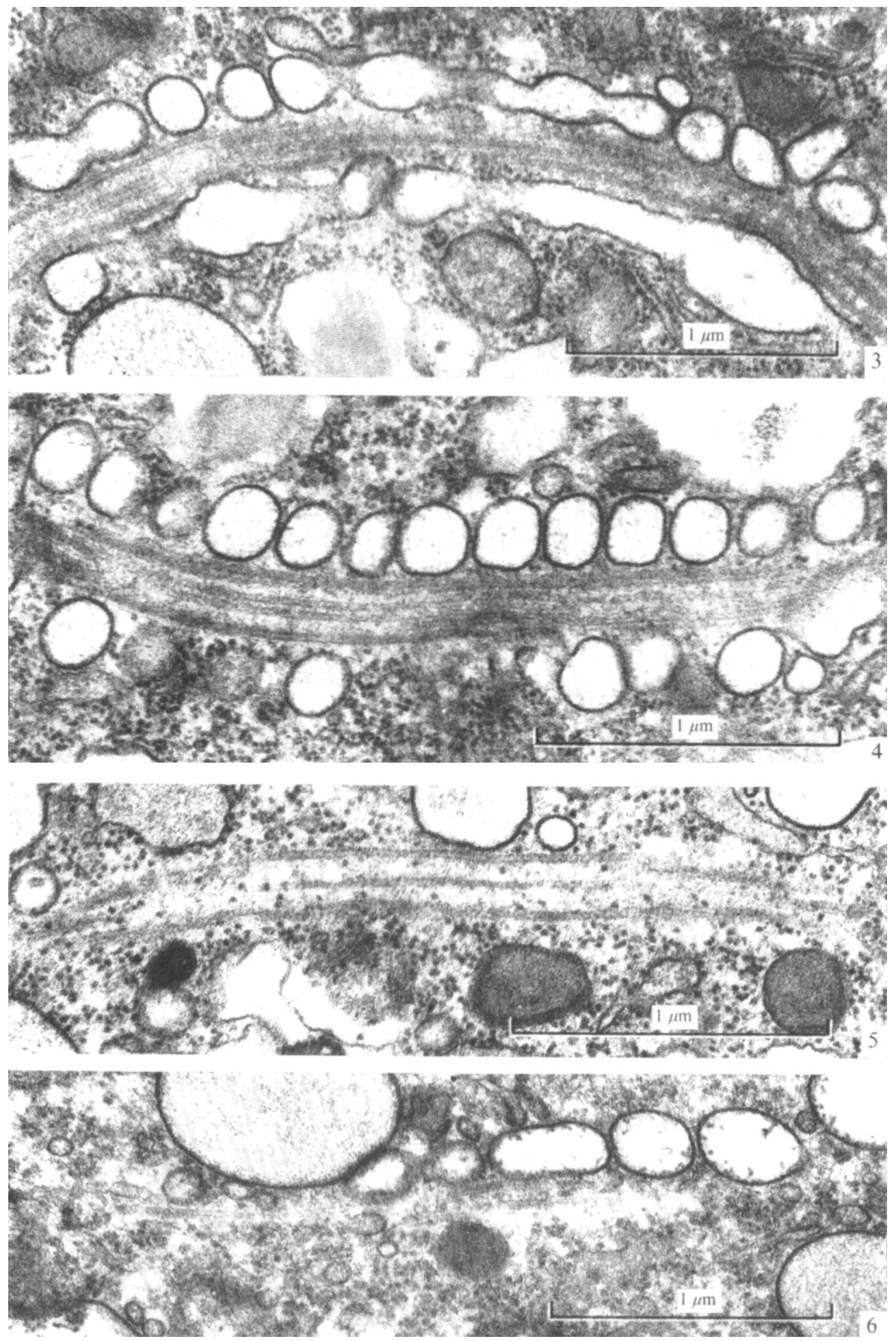

Fig. 3. Initial stages of cleavage of the flagellar vacuole (after $60 \mathrm{~min}$ of incubation).

Fig. 4. Vesicles coating flagellar axoneme immediately after vacuolar cleavage (after $60 \mathrm{~min}$ of incubation).

Fig. 5. Flagellar axoneme lying unenclosed in cytoplasm (after $90 \mathrm{~min}$ of incubation).

Fig. 6. Longitudinal section of an axoneme at a late stage of breakdown (after 105 min of incubation). 
For electron microscopy samples were taken at $15 \mathrm{~min}$ intervals until $180 \mathrm{~min}$ after the onset of incubation. The Petri dishes were decanted and then flooded with $5 \mathrm{ml}$ of $2 \%$ glutaraldehyde in $0 . \mathrm{I} \mathrm{M}-\mathrm{Na}$-cacodylate buffer, $\mathrm{pH} 7 \cdot 2$. After $\mathrm{I} h$ the suspensions were centrifuged at low speeds, embedded in $2 \%$ agar, washed eight to ten times in $0 . \mathrm{I} \mathrm{M}-\mathrm{Na}$ cacodylate buffer, $\mathrm{pH} 7 \cdot 2$, over a period of $30 \mathrm{~min}$, and postfixed in $\mathrm{I} \%$ osmium tetroxide in $0 \cdot \mathrm{I}$ M-Na-cacodylate buffer, $\mathrm{pH} 7 \cdot 2$, for $\mathrm{I} \mathrm{h}$, a fixation schedule described by Grove \& Bracker (1970). The specimens were again washed in Na-cacodylate buffer eight to ten times, dehydrated in ethanol and embedded in Epon 8I2. Thin sections were stained with lead citrate (Reynolds, 1963) and viewed in a Hitachi HU-I I E electron microscope.

\section{RESULTS}

Under the cultural conditions employed in this study, flagella were formed during sporangial development and were observed in $99 \%$ of the fully expanded sporangia. The flagella extended from paired basal bodies, located at the pointed ends of the pyriform nuclei, into vacuoles which ramified throughout the peripheral sporangial cytoplasm (Fig. I, 2, 7).

In material fixed after 15,30 and 45 min of incubation in dextrin, no ultrastructural changes could be observed. At $60 \mathrm{~min}$, however, striking alterations took place. The membranes of the flagellar vacuoles became undulated giving the vacuole a beaded appearance. This beaded appearance became progressively more pronounced and eventually the large vacuole broke up leaving the flagellar axonemes surrounded by chains of vesicles and unenclosed in the cytoplasm (Fig. 3, 4, 8, 9). Later these vesicles dispersed in the cytoplasm. Larger uncleaved segments of the vacuole occasionally assumed bell shapes and thus enclosed, and eventually completely isolated, segments of the cytoplasm. After $105 \mathrm{~min}$ of incubation naked axonemes were observed without any vesicular association (Fig. 5, IO, II). At this time two degenerative changes in the axoneme were observed: some of the central microtubules were missing (Fig. IO, II), and the rigid alignment of the peripheral microtubules was not preserved, so that they did not remain strictly parallel to each other (Fig. 6). In samples taken after I 20 min no traces of flagellar axonemes could be observed. However, the bundles of microtubules located in segments of the endoplasmic reticulum, which have been shown to become flagellar hairs (flimmer) in Saprolegnia, Dictyuchus, Synura and Cryptomonas by Heath, Greenwood \& Griffiths (1970) and in Pythium by Bracker, Heintz \& Grove (1970), were not broken down and were still observed in directly germinating sporangia.

Samples of sporangia, incubated as usual but in the presence of $10^{-5} \mathrm{~g}$ cycloheximide (Actidione)/ml, did not show signs either of flagellar degeneration or germination after $4 \mathrm{~h}$ when all the controls had germinated with germ tubes. The sporangial ultrastructure remained indistinguishable from that of resting sporangia.

Fig. 7. Transverse section of flagellum within flagellar vacuole in fully expanded sporangium.

Fig. 8. Vacuolar cleavage (after $60 \mathrm{~min}$ of incubation).

Fig. 9. Transverse section of naked axoneme after vacuolar cleavage (after 60 min of incubation).

Figs. I0, I I. Two examples of axonemal degeneration where one of the central pair of axonemal microtubules is missing (after $105 \mathrm{~min}$ of incubation).

Fig. I2. Flagellum entrapped within localized deposits of wall-like material. Note the accumulation of vesicles at the sporangial periphery. S, Original sporangial wall. 

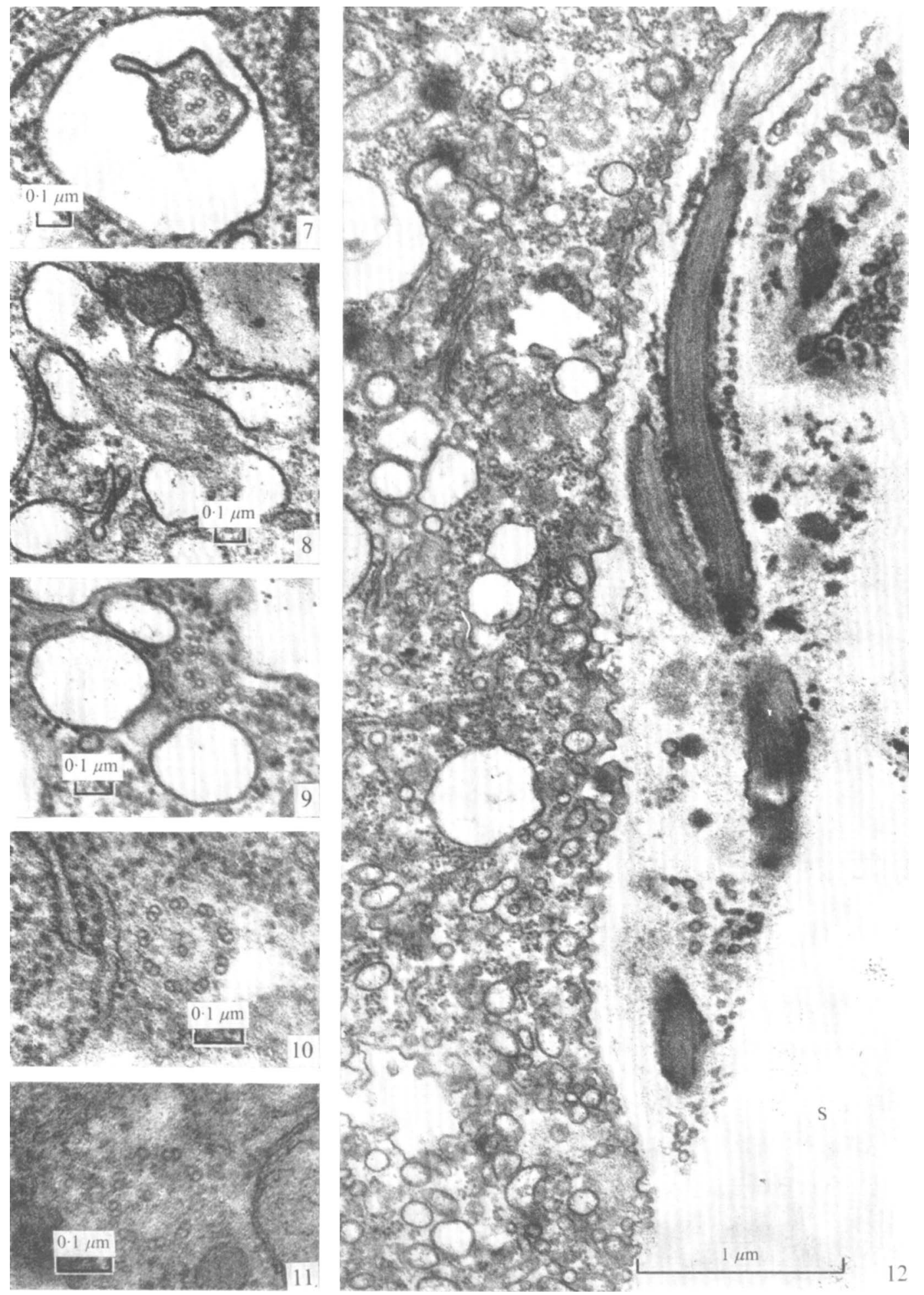
A second type of flagellar degeneration was observed in all samples taken during the germination period, but involved only a few flagella in approximately $5 \%$ of the sporangia. In these cases flagella became entrapped in localized deposits of wall-like material at the sporangial periphery (Fig. I2). This wall-like material appeared similar to that of the germination wall which is formed after $3 \mathrm{~h}$ of incubation (Hemmes \& Hohl, 1969). Accumulations of small vesicles $\mathrm{I} 50$ to $250 \mathrm{~nm}$ in diameter with amorphous contents were observed at the area of wall formation. The entrapped flagella usually appeared dense and in various stages of degeneration.

\section{DISCUSSION}

Sporangial germination in Phytophthora provides us with an interesting morphogenetic phenomenon: independent of the mode of germination, the sporangia always develop flagella (Hemmes \& Hohl, 1969). This is taken to indicate that the sporangium is not programmed to form either zoospores or germ tubes according to some selective trigger mechanism, but always develops in the direction of germination with zoospores and then reverses its trend when conditions favour germ-tube formation. This situation necessitates a mechanism which is capable of breaking down, upon a certain signal, the cell components unnecessarily formed. The occurrence of such a regular series of events leading to the complete breakdown of all flagella in directly germinating sporangia has been substantiated in this study. We therefore suspect that this organism possesses not only machinery programmed to form the flagellar apparatus but also an equally efficient system for controlled elimination of flagella under conditions favouring germination with germ tubes. Further evidence for controlled elimination of superfluous cell material has been obtained from other micro-organisms (Hohl, Hamamoto \& Hemmes, I968; Hohl \& Hamamoto, I969).

The breakdown of the flagella involves two main stages: first, the integrity of the membrane covering the flagellum is destroyed by cleavage of the ensheathing vacuole into small vesicles. By this mechanism the axoneme is left unenclosed in the cytoplasm. The removal of the ensheathing membrane appears to be an essential step in flagellar degeneration, since a comparable denuding takes place during flagellum retraction accompanying encystment of fungal zoospores where sections through naked axonemes in the cytoplasm have been observed (Umphlett \& Olson, I967; Cantino, Truesdell \& Shaw, 1968; Fuller \& Reichle, I968; Koch, I968; Reichle, I969; Lesemann \& Fuchs, I970; Heath \& Greenwood, I971). We assume that in all these cases the flagella are in a stage of degeneration.

In the second stage the axonemes gradually disintegrate whereby two processes, a gradual loosening of the parallel alignment of the microtubules and a depolymerization of the microtubules, can be disinguished. Our observations show that the central microtubules break down first and thus appear to be more fragile than the peripheral ones as has been suggested previously by Roth \& Shigenaka (1964) for cilia of Diplodinium ecaudatum and by Grimstone \& Klug (1966) for isolated flagella of Trichonympha. In a final stage all microtubules have disappeared. This indicates to us that, at least in direct sporangial germination of Phytophthora palmivora, neither intact flagella nor flagellar microtubules are preserved to help in the construction of future zoospores, which has been suggested by Reichle (1969) for the case of repeated zoospore emergence in Phytophthora parasitica var. nicotiana. 


\section{REFERENCES}

Aragaki, M. \& Hine, R. B. (1963). Effects of radiation on sporangial production of Phytophthora parasitica on artificial media and detached papaya fruit. Phytopathology 53, 854-856.

Bracker, C. E., Heintz, C. E. \& Grove, S. N. (1970). Structural and functional continuity among endomembrane organelles in fungi. In Résumés des communications présentées au septiéme congrès international, Grenoble, France, vol. III, pp. 103-104. Edited by P. Favard. Paris: Société C.I.B.

Cantino, E. D., Truesdell, L. C. \& Shaw, D. S. (1968). Life history of the motile spore of Blastocladiella emersonii: a study in cell differentiation. Journal of the Elisha Mitchell Scientific Society 84, I25-146.

Fuller, M.S. \& ReIChle, R. E. (1968). The fine structure of Monoblepharella sp. zoospores. Canadian Journal of Botany 46, 279-283.

Grimstone, A. V. \& KLUG, A. (1966). Observations of the substructure of flagellar fibres. Journal of Cell Science I, 351-362.

Grove, S. N. \& Bracker, C. E. (1970). Protoplasmic organization of hyphal tips among fungi: Vesicles and Spitzenkörper. Journal of Bacteriology ro4, 989-1009.

HeATH, I. B. \& GreEnwood, A. D. (197I). Ultrastructural observations on the kinetosomes and golgi bodies during the asexual life cycle of Saprolegnia. Zeitschrift fïr Zellforschung und mikroskopische Anatomie 112, 371-389.

Heath, I. B., Greenwood, A. D. \& Griffiths, H. B. (1970). The origin of flimmer in Saprolegnia, Dictyuchus, Synura and Cryptomonas. Journal of Cell Science 7, 445-46I.

Hemmes, D. E. \& Hohl, H. R. (I969). Ultrastructural changes in directly germinating sporangia of Phytothora parasitica. American Journal of Botany 56, 300-313.

Hohl, H. R. \& Намамото, S. T. (1969). Ultrastructure of spore differentiation in Dictyostelium: The prespore vacuole. Journal of Ultrastructure Research 26, 442-453.

Hohl, H. R., Намамото, S. T. \& Hemmes, D. E. ( I 968). Ultrastructural aspects of cell elongation, cellulose synthesis and spore differentiation in Acytostelium leptosomum, a cellular slime mold. American Journal of Botany 55, 783-796.

Kосн, W. J. (I968). Studies of the motile cells of chytrids. V. Flagellar retraction in posteriorly uniflagellate fungi. American Journal of Botany 55, $841-859$.

LesemanN, D. E. \& Fuchs, W. H. (1970). Elektronenmikroskopische Untersuchung über die Vorbereitung der Infektion in encystierten Zoosporen von Olpidium brassicae. Archiv für Mikrobiologie 7r, 9-19.

REICHLE, R. E. (I969). Retraction of flagella by Phytophthora parasitica var. nicotiana zoospores. Archiv für Mikrobiologie 66, 340-347.

REYNOLDS, E. S. ( I963). The use of lead citrate at high $\mathrm{pH}$ as an electron-opaque stain in electron microscopy. Journal of Cell Biology 17, 208-2 I 2.

Roth, L. E. \& ShigenaKa, Y. (1964). The resorption of cilia. Zeitschrift für Zellforschung und mikroskopische Anatomie 64, 19-24.

TOKunAGA, J. \& BARTNICKI-GARCIA, S. (1971). Cyst wall formation and endogenous carbohydrate utilization during synchronous encystment of Phytophthora palmivora zoospores. Archiv für Mikrobiologie 79, 283-292.

Umphlett, C. J. \& Olson, L. W. (1967). Cytological and morphological studies on a new species of Phlyctochytrium. Mycologia 59, $1085-1096$. 